\title{
BMJ Open Effectiveness of combined vaginal progesterone and cervical cerclage in preventing preterm birth: a systematic review and meta-analysis protocol
}

\author{
Rosanna C Diacci (D) , ${ }^{1}$ Ashad Issah, ${ }^{1}$ Kimberley P Williams, ${ }^{1}$ Liam McAuliffe, ${ }^{1}$ \\ Anne-Marie Aubin, ${ }^{1}$ Jack E McAuliffe, ${ }^{2}$ Jason Phung, ${ }^{1,3}$ Carol A Wang, ${ }^{1,3}$ \\ Craig E Pennell ${ }^{1,3}$
}

To cite: Diacci RC, Issah A, Williams KP, et al. Effectiveness of combined vaginal progesterone and cervical cerclage in preventing preterm birth: a systematic review and metaanalysis protocol. BMJ Open 2021;11:e050086. doi:10.1136/ bmjopen-2021-050086

- Prepublication history and additional supplemental material for this paper are available online. To view these files, please visit the journal online (http://dx.doi.org/10.1136/ bmjopen-2021-050086)

Received 10 February 2021 Accepted 24 May 2021

Check for updates

(C) Author(s) (or their employer(s)) 2021. Re-use permitted under CC BY-NC. No commercial re-use. See rights and permissions. Published by BMJ.

${ }^{1}$ School of Medicine and Public Health, College of Health, Medicine and Wellbeing, The University of Newcastle, Callaghan, New South Wales, Australia

${ }^{2}$ Department of Engineering, Computing and Mathematical Sciences, University of Adelaide, Adelaide, South Australia,

Australia

${ }^{3}$ Mothers and Babies Research Centre, Hunter Medical Research Institute, New Lambton, New South Wales, Australia

Correspondence to

Professor Craig E Pennell;

craig.pennell@newcastle.edu.au

\section{ABSTRACT}

Introduction Preterm birth (PTB) is the leading cause of death in children under 5 years. Preventive therapies targeted towards women with risk factors such as a prior PTB or a short cervix reduce the rate of PTB. Cervical cerclage, vaginal progesterone and a combination of the two have been used with no consensus as to whether combined treatment is more effective than any single treatment alone. The objective of this review is to determine the efficacy of combined treatment compared with cerclage alone and combined treatment compared with progesterone alone.

Methods and analysis Studies will be sourced from the electronic databases Medline (Ovid), EMBASE (Ovid), PsycINFO (Ovid), Scopus, CINAHL (EBSCOhost) and Cochrane Library (Wiley) and reference lists. We will not exclude any papers due to publication date. Randomised control trials (RCTs), non-RCTs and cohort studies assessing single therapy (either progesterone or cerclage) versus combined therapy in women with a singleton pregnancy will be included. Two independent reviewers will conduct study screening (at abstract and full-text level), data extraction and risk of bias assessment with disagreements resolved by an experienced researcher. Random or fixed effects models will be used depending on data heterogeneity and data will be presented as risk ratio for dichotomous data or mean difference for continuous data with a $\mathrm{Cl}$ of $95 \%$ used for all outcomes.

Ethics and dissemination Not applicable due to nature of the study type.

PROSPERO registration number CRD42020195975.

\section{INTRODUCTION}

Preterm birth (PTB), defined as birth before 37 weeks, ${ }^{1}$ occurs in 5\%-13\% of all pregnancies. ${ }^{2}$ It is associated with neonatal mortality and is the leading cause of death in children less than 5 years, ${ }^{3}$ as well as significant neonatal morbidity such as infant respiratory distress syndrome, intraventricular haemorrhage, necrotising enterocolitis and retinopathy of prematurity. ${ }^{4}$
Strengths and limitations of this study

- The systematic review will follow the rigorous methods outlined in this protocol which have been written as per Cochrane guidelines.

- This will be the first systematic review to answer this question.

- Data will be screened and extracted by two reviewers.

- Lack of reviewer and moderator blinding at inclusion/exclusion level.

- Lack of blinding of reviewers and moderators for papers at quality assessment Risk of Bias in NonRandomised Studies of Interventions tool, Risk of Bias in Randomised Studies of Interventions tool and Grade of Recommendation, Assessment, Development and Evaluations.

The majority of PTB occurs either spontaneously or following preterm premature rupture of membranes (PPROM) ${ }^{5}$ It is well established that a cervical length of less than $25 \mathrm{~mm}$, measured between 18 and 25 weeks, is a good predictor of spontaneous PTB (sPTB) with rates of $31 \%-41 \% .^{67}$ Vaginal progesterone $^{8}$ and cervical cerclage ${ }^{69}$ are effective single treatments for the prevention of sPTB in these women, as well as those with a prior history of PTB.

Cervical cerclage is a treatment proven to prevent PTB and reduce neonatal morbidity and mortality ${ }^{10-12}$ by mechanically maintaining a long and closed cervix. In contrast, progesterone has an inhibitory action on uterine contractility by inhibiting the production of stimulatory prostaglandins and expression of contraction associated protein genes in the myometrium. ${ }^{13} 14$ It has been shown to play an important role in maintaining a pregnancy until term. ${ }^{15}$ 
More recently, studies have assessed the combination of the cervical cerclage and vaginal progesterone to improve PTB prevention. ${ }^{10}{ }^{11}$ To our knowledge, only one systematic review published in 2013 has addressed progesterone as an adjunctive therapy to cerclage; however, the included studies were not randomised and assessed synthetic progestin 17-hydroxyprogesterone caproate, which found no difference in the outcome of PTB. ${ }^{13}$ To our knowledge, there has not been any systematic review addressing combined treatment of progesterone and cerclage versus singular treatment since $2017 ;{ }^{16}$ with no review specifically assessing vaginal progesterone in combined treatment. More recently, adjuvant vaginal progesterone therapy for women who underwent cervical cerclage indicated by ultrasound ${ }^{11}$ or physical ${ }^{10}$ examination was found to be associated with decreased rates of PTB and admission to the neonatal intensive care unit (NICU). Given these recent promising findings and the lack of guidance on this topic, we sought to determine the effect of combining both cerclage and progesterone on PTB by conducting a systematic review and metaanalysis. This paper describes the proposed protocol for this meta-analysis.

\section{AIM}

This systematic review has two aims: (1) to compare the use of cerclage alone to cerclage and vaginal progesterone combined and (2) to compare progesterone alone to the combined use of cerclage and vaginal progesterone in order to determine which of these are associated with better maternal and neonatal outcomes in relation to PTB. Our proposed review will answer the questions: in women requiring prophylactic treatment for short cervix, is combined treatment favourable to cerclage alone? And in women requiring prophylactic treatment for short cervix, is combined treatment favourable to vaginal progesterone alone?

\section{METHODS}

\section{Registration}

This systematic review protocol was submitted to the International Prospective Register of Systematic Reviews on 8 October 2020 and was last updated on this date. This review and meta-analysis will be completed in accordance with the recommendations of both Preferred Reporting Items for Systematic Reviews and Meta-Analyses Protocol ${ }^{17}$ and the Cochrane Handbook for Systematic Reviews of Interventions. $^{18}$

Information regarding registration can be accessed from http://wwwcrdyorkacuk/PROSPERO.

\section{Eligibility criteria}

The eligibility of studies included will be based on inclusion and exclusion criteria applied to the domains of participant, exposure, comparator, study type and outcome.

\section{Participants}

The review will consider all studies which include women who are undergoing ultrasound or whose history indicated cerclage, vaginal progesterone or both for the prevention of PTB. Only singleton pregnancies will be assessed.

\section{Intervention}

Studies comparing combined cerclage and vaginal progesterone treatment with vaginal progesterone alone or cerclage alone.

\section{Cerclage}

There are two commonly performed vaginal cerclage procedures which were first described by Shirodkar and McDonald. In the McDonald approach, a suture is placed around the cervix in purse-string fashion and securely tied anteriorly. The McDonald approach requires no dissection into paracervical tissues. ${ }^{19} 20$ The Shirodkar technique involves a transverse anterior colpotomy, dissection of the bladder up to the internal cervical os and a posterior colpotomy with dissection of peritoneum upwards to the internal os. The suture is placed subcutaneously and the knot is tied in the posterior defect and buried under the vaginal epithelium ${ }^{19} 2122$ or is tied exterior to the epithelium in the modified approach. Both Shirodkar techniques will be considered.

\section{Vaginal progesterone}

Vaginal progesterone is available as a gel, suppository or pessary. ${ }^{14}$ It is the most bioavailable form of progesterone for uterine and cervical effects with the fewest side effects. Its micronised form decreases particle size and increases surface area resulting in improved absorption with less metabolic and vascular side effects. ${ }^{23}$ The vaginal route also allows rapid absorption and avoids first-pass hepatic metabolism, resulting in high bioavailability in the uterus. ${ }^{24}$

\section{Combined treatment}

Cervical cerclage (McDonald or Shirodkar technique) used in combination with vaginal progesterone.

\section{Outcomes}

The primary outcome is PTB, defined as birth $<37$ weeks gestation. Secondary dichotomous outcomes will be PTB $<34$ weeks, <32 weeks and <28 weeks; PPROM; caesarean section and neonatal complications: NICU admission, intubation and neonatal mortality. The continuous secondary outcomes will be gestational age at delivery; birth weight and number of days between intervention and delivery.

\section{Types of studies}

The review will include randomised and pseudorandomised control trials, non-randomised experimental control trials and cohort studies. All included papers must compare cerclage to combined treatment and/or vaginal progesterone to combined treatment. Those studies which also presented a control group will be included. 


\section{Search strategy}

Electronic bibliographic databases will be searched for eligible, peer-reviewed literature including: Medline (Ovid), EMBASE (Ovid), PsycINFO (Ovid), Scopus, CINAHL (EBSCOhost) and Cochrane Library (Wiley). Reference lists of included studies will also be screened for eligible papers. Studies recommended by experts, the references of textbooks and grey literature will also be reviewed for this purpose. We will place no restriction on the length of study follow-up time or on country, year or language of publication. All studies will be human trials. See online supplemental appendix 1.

The search strategy will be developed through discussion with experts and academics, pilot searches and by assessing systematic reviews with similar questions. The search strategy will focus on identifying relevant interventions with no population or outcome-related keywords used. The intervention search terms will be: cervical OR cervix OR rescue; stitch OR cerclage OR suture; progesterone OR progestin OR prometrium OR progest. Medical subject headings will be used when relevant and present databases.

\section{Data collection and analysis}

Study selection

Identified titles and abstracts will be downloaded into Endnote $^{25}$ where duplicated studies will be removed. Remaining papers will then be uploaded to Covidence ${ }^{26}$ and then screened against the eligibility criteria outlined above. Full texts of remaining studies will be sourced and screened before undergoing critical appraisal and data extraction. All screening will be performed by two independent reviewers and any disagreements will be addressed by a senior research moderator. No reviewers or moderators will be blinded to titles, authors, journals or institutions.

\section{Data management}

The search will be uploaded to Covidence,${ }^{26}$ an internetbased software which allows collaboration between multiple reviewers during the study selection process, backup copies of all studies will also be kept in an Endnote library. ${ }^{25}$

\section{Data collection}

Two reviewers will extract data through Covidence ${ }^{26}$ using a standardised electronic form consistent with data collection items recommended by the Cochrane Handbook for Systematic Reviews of Interventions. ${ }^{18}$ This process will be piloted prior to use and any discrepancies will be moderated by a third senior research moderator. Once extracted, on reviewer agreement, data will be transferred from Covidence ${ }^{26}$ into Review Manager dataanalysis software. ${ }^{27}$

The following data will be extracted:

- Study characteristics: authors; publication date; study design; country of study; sample size; confounding factors of participants; publication status; trial size; funding and risk of bias information.

- Intervention characteristics: type of intervention used; reason for intervention; patient characteristics (maternal age, gravity, parity) and any cointerventions received.

- Outcomes: maternal, fetal and neonatal outcome data and definitions of each of the outcomes as described below.

\section{Outcomes and prioritisation \\ Primary outcome}

PTB defined as live birth or stillbirth with a gestational age between 20 and 37 weeks. Primary outcome is birth $<37$ weeks gestation with subanalysis at $<34$ weeks, $<32$ weeks and $<28$ weeks.

For outcomes which report these data as 'greater than' $\mathrm{X}$ weeks gestation, data extractors will manually invert the figure to less than.

\section{Secondary outcomes}

\section{Dichotomous}

1. PPROM

2. Caesarean section.

3. NICU admission.

4. Intubation.

5. Neonatal mortality.

\section{Continuous}

1. Gestational age at delivery.

2. Birth weight.

3. Number of days between intervention and delivery.

\section{Assessment of risk of bias}

Each paper will be assessed for risk of bias using the Cochrane Collaboration tool for assessing risk of bias (Risk of Bias in Non-Randomised Studies of Interventions (ROBINS-I) and Risk of Bias in Randomised Studies of Interventions (RoB-2)) ${ }^{28}{ }^{29}$ Each study will be reviewed independently by two assessors and disagreements will be resolved through mediation with a third reviewer. Highquality studies are those which achieve a score of 7 or 8 , average are scored $4-6$ and below 4 will be considered low quality. We will not be excluding any study based on these scores, however risk of bias will be taken into account when outcomes are assessed in regard to impact as per the Grade of Recommendation, Assessment, Development and Evaluations (GRADE). ${ }^{30}$

The ROBINS-I tool ${ }^{28}$ will be used to assess the risk of bias in included observational studies. The risk of bias will be rated as no information, low risk, moderate risk, serious risk or critical risk across seven domains. The seven domains of this tool are (1) confounding; (2) selection of participants; (3) classification of intervention; (4) deviation from interventions; (5) missing outcome data; (6) measurement of outcomes and (7) selection of reported results overall.

Randomised trials will be assessed with the RoB-2. ${ }^{28}$ This tool assesses five domains which are: (1) the randomisation 
proces; (2) deviations from intended intervention; (3) missing outcome data; (4) measurement of the outcome and (5) selection of the reported results. Studies which score a high risk of bias in one or more domains or which have concerns for several domains will be judged as at serious risk of bias.

\section{Cochrane GRADE assessment}

The GRADE tool will be used to assess quality of evidence for the primary outcome. ${ }^{30}$ The outcome will be assessed in terms of bias risk, consistency, directness, precision and publication bias. The primary outcome's quality will be judged to be (1) high quality—we are very confident that the true effect is close to that of the estimated effect; (2) moderate-it is possible that there is a substantial difference but we are moderately confident that the true effect is close to that of the estimated effect; (3) low-we are limited in our confidence that the estimated effect and true effect reflect each other and (4) very low-we have very little confidence that the true and estimated effect align, the true effect is likely to be substantially different from our estimate. GRADE will be conducted by two independent reviewers and discrepancies will be resolved through discussion, disagreements which cannot be resolved will be mediated by a third reviewer.

Graphic representations of potential bias within and across the studies will be calculated using Review Manager V.5.3 (RevMan V.5.3) ${ }^{27}$ All items in the risk of bias assessment will be considered independently without an attempt to collate and assign an overarching score.

\section{Data synthesis}

Meta-analysis will be constructed by pooling studies using Covidence $^{26}$ and RevMan V.5.3 software. ${ }^{27}$ Forest plots and $\mathrm{I}^{2}$ values will be used to explore the heterogeneity of data. Heterogeneity of data will be examined using forest plots and quantified throughout using the calculation of the $I^{2}$ value. A random effects model will be used when a data set meets three of the following four criteria: (1) there is an intention to use results beyond the included studies, (2) number of included studies greater than five, (3) there is statistical heterogeneity measured as an $\mathrm{I}^{2}$ greater or equal to $50 \%$ and (4) it is reasonable to assume that included studies estimate a different underlaying true effect with normal distribution. ${ }^{31}$ If a data set does not meet three or more criteria, a fixed effects model will be used. All outcomes for which a random effects model is used will undergo a second examination using the Hartung-Knapp-Sidik-Jonkman method for random effects to ensure considerable heterogeneity does not impact the data. For reporting consistency between outcomes, the monotherapy (cerclage or progesterone) intervention was made the reference set for all analyses, standardising the direction of effect across all primary and secondary outcomes.

\section{Measures of treatment effect}

Dichotomous outcomes will be assessed and reported using risk ratios, while continuous data will be reported using mean difference (MD) or standardised MD (SMD), 95\% CIs will be used for all data sets. An SMD will be used when studies report a comparable but not identical measure for the same outcome. To avoid discarding important data from papers that do not report the mean and SD of continuous data, we will attempt to calculate means and SDs using known parameters. For papers which reported median and range, Hozo $e$ e $a l$ 's approach will be used. ${ }^{32}$ For papers which reported median and IQR, the Wan et al's approach will be employed. ${ }^{33}$ Data that are too positively or negatively skewed render the mean and SD unsuitable for these approaches, particularly when the SD is large. ${ }^{34}$ For this reason, data which are not suitable to be estimated with mean and SD will be excluded as per the Cochrane Handbook. $^{34}$ Where meta-analysis is not possible, alternative synthesis methods, including summarising effect estimates and combining $\mathrm{p}$ values, will be used as recommended by the Cochrane Handbook for Systematic Reviews of Interventions. ${ }^{18}$

\section{Missing data}

For studies which presented missing data we will attempt to contact authors. However, if this is not possible, we will conduct sensitivity analysis which will exclude trials with $>30 \%$ missing data. ${ }^{18}$

\section{Meta-bias(es)}

To determine reporting bias, we will attempt to investigate to see if protocols for included studies were published prior to those studies being started.

\section{Sensitivity analysis}

Sensitivity analysis will be conducted on the primary outcome for birth $<37$ weeks gestation for dual interventions (cerclage vs combined and progesterone vs combined). This will be done by removing studies which are judged to have an overall critical risk of bias, allowing us to examine their impact on the effect estimate of the primary outcome.

Additional sensitivity analysis will be conducted excluding studies which assessed combined therapy in a sequential manner. For this sensitivity analysis, we will define 'stepwise' as cerclage that is placed $>14$ days following the failure of progesterone to prevent further cervical shortening, or as progesterone that is initiated $>14$ days following the failure of cerclage wherein the initial intervention has been ineffective in preventing cervical shortening.

Where individual patient data are available, neonatal outcomes and baseline maternal characteristics will be extracted from studies and subanalysis will be conducted if vast differences are found in these baseline characteristics. 


\section{DISCUSSION}

This systematic review and meta-analysis will determine the differences in effectiveness of cerclage alone versus combined treatment, as well as the differences in effectiveness between progesterone alone versus combined treatment. These results will provide valuable synthesis of information to specialists in their clinical decisions for women at risk of PTB. It is hoped that women at high risk of sPTB and its complications benefit from these findings. The results of this paper could potentially bring updates to clinical management guidelines and reduce the shortterm and long-term negative health outcomes of PTB for women and their children.

Acknowledgements The authors would like to thank Ms Joanne Davies for her editing and advice.

Contributors RCD, Al, KPW, LM, A-MA and JEM: led the development of this manuscript. CW and JEM: provided guidance on the statistical plan. JP and CP: provided expertise relating to obstetric care. All authors: read and approved the final manuscript, conception and design, acquisition of data or analysis, interpretation of data and outlined the study design. RCD, A-MA, KPW, LM and CP: drafted the article and discussion or revised it critically for important intellectual content (drafted introduction, aim and abstract). Al, JEM, JP, CW and CP: drafted methods.

Funding The authors have not declared a specific grant for this research from any funding agency in the public, commercial or not-for-profit sectors.

Competing interests JP is supported by the Hunter New England Health Clinical and Health Research Fellowship.

Patient and public involvement statement Patients and/or the public were not involved in the design, or conduct, or reporting, or dissemination plans of this research.

\section{Patient consent for publication Not required.}

Provenance and peer review Not commissioned; externally peer reviewed.

Supplemental material This content has been supplied by the author(s). It has not been vetted by BMJ Publishing Group Limited (BMJ) and may not have been peer-reviewed. Any opinions or recommendations discussed are solely those of the author(s) and are not endorsed by BMJ. BMJ disclaims all liability and responsibility arising from any reliance placed on the content. Where the content includes any translated material, BMJ does not warrant the accuracy and reliability of the translations (including but not limited to local regulations, clinical guidelines, terminology, drug names and drug dosages), and is not responsible for any error and/or omissions arising from translation and adaptation or otherwise.

Open access This is an open access article distributed in accordance with the Creative Commons Attribution Non Commercial (CC BY-NC 4.0) license, which permits others to distribute, remix, adapt, build upon this work non-commercially, and license their derivative works on different terms, provided the original work is properly cited, appropriate credit is given, any changes made indicated, and the use is non-commercial. See: http://creativecommons.org/licenses/by-nc/4.0/.

\section{ORCID iD}

Rosanna C Diacci http://orcid.org/0000-0002-6862-4042

\section{REFERENCES}

1 WHO: recommended definitions terminology format for statistical tables related to the perinatal period use of a new certificate for cause of perinatal deaths. Modifications recommended by FIGO as amended October 14, 1976. Acta Obstetricia et Gynecologica Scandinavica 1977;56:247-53.

2 Blencowe H, Cousens S, Oestergaard MZ, et al. National, regional, and worldwide estimates of preterm birth rates in the year 2010 with time trends since 1990 for selected countries: a systematic analysis and implications. Lancet 2012;379:2162-72.

3 Chawanpaiboon S, Vogel JP, Moller A-B, et al. Global, regional, and national estimates of levels of preterm birth in 2014: a systematic review and modelling analysis. Lancet Glob Health 2019;7:e37-46.
4 Ward RM, Beachy JC. Neonatal complications following preterm birth. BJOG 2003;110(Suppl 20):8-16.

5 Goldenberg RL, Culhane JF, lams JD, et al. Epidemiology and causes of preterm birth. The Lancet 2008;371:75-84.

6 Berghella V, Rafael TJ, Szychowski JM, et al. Cerclage for short cervix on ultrasonography in women with singleton gestations and previous preterm birth: a meta-analysis. Obstet Gynecol 2011;117:663-71.

7 Souza RT, Costa ML, Mayrink J, et al. Clinical and epidemiological factors associated with spontaneous preterm birth: a multicentre cohort of low risk nulliparous women. Sci Rep 2020;10:1-10.

8 Romero R, Conde-Agudelo A, Da Fonseca E, et al. Vaginal progesterone for preventing preterm birth and adverse perinatal outcomes in singleton gestations with a short cervix: a metaanalysis of individual patient data. Am J Obstet Gynecol 2018;218:161-80.

9 MRC/RCOG Working Party on Cervical Cerclage. Final report of the medical research Council/Royal College of obstetricians and gynaecologists multicentre randomised trial of cervical cerclage. MRC/RCOG Working Party on cervical Cerclage. Br J Obstet Gynaecol 1993;100:516-23.

10 Jung EY, Oh KJ, Hong J-S, et al. Addition of adjuvant progesterone to physical-exam-indicated cervical cerclage to prevent preterm birth. J Obstet Gynaecol Res 2016;42:1666-72.

11 Park JY, Jung YM, Kook S-Y, et al. The effect of postoperative vaginal progesterone in ultrasound-indicated cerclage to prevent preterm birth. J Matern Fetal Neonatal Med 2019:1-8.

12 Beck S, Wojdyla D, Say L, et al. The worldwide incidence of preterm birth: a systematic review of maternal mortality and morbidity. Bull World Health Organ 2010;88:31-8.

13 Defranco EA, Valent AM, Newman T, et al. Adjunctive therapies to cerclage for the prevention of preterm birth: a systematic review. Obstet Gynecol Int 2013;2013:1-10.

14 Sfakianaki AK, Norwitz ER. Mechanisms of progesterone action in inhibiting prematurity. J Matern Fetal Neonatal Med 2006;19:763-72.

15 Kuon R-J, Voß P, Rath W. Progesterone for the Prevention of Preterm Birth - an Update of Evidence-Based Indications. Geburtshilfe Frauenheilkd 2019;79:844-53.

16 Jarde A, Lewis-Mikhael A-M, Dodd JM, et al. The more, the better? Combining interventions to prevent preterm birth in women at risk: a systematic review and meta-analysis. J Obstet Gynaecol Can 2017;39:1192-202.

17 Moher D, Shamseer L, Clarke M, et al. Preferred reporting items for systematic review and meta-analysis protocols (PRISMA-P) 2015 statement. Syst Rev 2015;4:1.

18 Higgins J, Thomas J. Cochrane Handbook for systematic reviews of interventions version 6.0 (updated July 2019). Chandler J CM, Li T, Page MJ, Welch VA editor, 2019.

19 Jones HRJ. Te Lindes operative gynecology. Lippincott, Williams \& Wilkins, 2015.

$20 \mathrm{McDonald} \mathrm{IA}$. Suture of the cervix for inevitable miscarriage. J Obstet Gynaecol Br Emp 1957;64:346-50.

21 Shirodkar V. A new method of operative treatment for habitual abortions in the second trimester of pregnancy. Antiseptic 1955;52:299-300.

22 Barter RH, Dusbabek JA, TYNDAL CM, et al. Further experiences with the Shirodkar operation. Am J Obstet Gynecol 1963;85:792-805.

23 de Lignieres B, Dennerstein L, Backstrom T. Influence of route of administration on progesterone metabolism. Maturitas 1995;21:251-7.

24 Cicinelli E, Schonauer LM, Galantino P, et al. Mechanisms of uterine specificity of vaginal progesterone. Hum Reprod 2000;15 Suppl 1:159-65.

25 Endnote X9. Clarivate analytics. Philadelphia, United States: Thomson Reuters, 2019.

26 Covidence systematic review software. Melbourne, Australia: Veritas Health Innovation

27 Manager R. (RevMan). [Computer program]. Copenhagen The Nordic Cochrane Centre: The Cochrane Collaboration, 2014.

28 Sterne JA, Hernán MA, Reeves BC, et al. ROBINS-I: a tool for assessing risk of bias in non-randomised studies of interventions. BMJ 2016;355:i4919.

29 Sterne JAC, Savović J, Page MJ, et al. RoB 2: a revised tool for assessing risk of bias in randomised trials. BMJ 2019;366:14898.

30 Schünemann HBJ, Guyatt G, Oxman A, eds. Handbook for grading the quality of evidence and the strength of recommendations using the GRADE approach (updated October 2013). GRADE Working Group, 2013. guidelinedevelopment.org/app/handbook/handbook. html 
31 Tufanaru C, Munn Z, Stephenson M, et al. Fixed or random effects meta-analysis? Common methodological issues in systematic reviews of effectiveness. Int J Evid Based Healthc 2015;13:196-207.

32 Hozo SP, Djulbegovic B, Hozo I. Estimating the mean and variance from the median, range, and the size of a sample. BMC Med Res Methodol 2005;5:13.
33 Wan X, Wang W, Liu J, et al. Estimating the sample mean and standard deviation from the sample size, median, range and/or interquartile range. BMC Med Res Methodol 2014;14:135.

34 Higgins J, Green S, eds. Medians and interquartile ranges. Cochrane Handbook for Systematic Reviews of Interventions Version 5.1.0. London: The Cochrane Collaboration, 2011. 